density (BMD) and T-scores in patients with rheumatoid arthritis (RA), compared to a control group.

Methods: We enrolled 150 menopausal women, 75 diagnosed with RA and 75 age matched controls. The controls were selected considering the lack of both an inflammatory disease and history of corticotherapy.

All patients in the study group were under monotherapy with a conventional synthetic DMARD and they are or have been under corticotherapy during the evolu tion of RA.

The BMD and T score were evaluated using a quantitative ultrasound Echolight machine. There were two evaluators for both lots, on order to minimise the interobserver variability.

Results:

Abstract AB1214 - Table 1

\begin{tabular}{lcc}
\hline & Study group & Control group \\
\hline Age distribution (years) & $63,24^{49-85}$ & $64,19^{41-85}$ \\
Menopause age (years) & $46,9(34-60)$ & $45,78^{30}-55$ \\
BMI (kg/m ${ }^{2}$ ) & $25.97(15.63-34.86)$ & $26.83(18.36-33.67)$ \\
Period since dg of RA (years) & $7.26(5-12.5)$ & - \\
\hline
\end{tabular}

$13 \%$ of the patients in the study group were under corticotherapy at the moment they were recruited in the study and $87 \%$ were treated with cortisone before, at some point during the evolution of RA. The average dose followed for more than 2 weeks was $8.8(5-15) \mathrm{mg}$ prednisone/day. The average corticotherapy period of $2.6(0.5-14)$ months.

For the lumbar vertebrae (L1-L4), the average $T$ score in the study group was -1.81 , while the control group had a T score of -1.11 . For the femoral neck, the average $\mathrm{T}$ score for both hips was -1.73 for the study group and -1.04 for the controls.

The spine average BMD was $0.92 \mathrm{~g} / \mathrm{cm}^{2}$ in the study group, compared to $1.16 \mathrm{~g} /$ $\mathrm{cm}^{2}$ in the control group. For the femoral neck, the study group average BMD was $0.72 \mathrm{~g} / \mathrm{cm}^{2}$, while in the control group it was $0.94 \mathrm{~g} / \mathrm{cm}^{2}$.

Conclusions: The differences between the two groups were significant, but still in the osteopenia interval. The significance of these results translates into an increased fracture risk and a longer treatment duration in the study group. At this point, this is a preliminary study, but we plan to continue it by comparing these results to DXA results for the same patients, in order to evaluate the costeffective superiority of this portable, radiation-free technique, in a screening programme.

REFERENCES:

[1] Casciaro S, Peccarisi M, Pisani P, et al. An Advanced Quantitative Echosound Methodology for Femoral Neck Densitometry. Ultrasound Med Biol 2016; 42(6):1337-1356.

[2] Aventaggiato $\mathrm{M}$, Conversano $\mathrm{F}$, Pisani $\mathrm{P}$, et al. Validation of an automatic segmentation method to detect vertebral interfaces in ultrasound images. IET Science, Measurement \& Technology 2016; 10(1):18 - 27.

Disclosure of Interest: None declared

DOI: 10.1136/annrheumdis-2018-eular.7408

\section{AB1215 MINIMAL INVASIVE ULTRASOUND-GUIDED PAROTID GLAND BIOPSY IN CADAVERS DONE BY RHEUMATOLOGISTS}

R. Micheroli ${ }^{1}$, U. Wagner ${ }^{2}$, M. Mueller-Gerbl ${ }^{3}$, M. Toranelli ${ }^{4}$, C. Marx ${ }^{5}$, G.A. W. Bruyn ${ }^{6}$, S. Jousse-Joulin ${ }^{7}$, G. Tamborrini $i^{5} .{ }^{1}$ Department of Rheumatology, University Hospital of Zurich, Zurich; ${ }^{2}$ Pathology, Unilabs Mittelland, Bern; ${ }^{3}$ Department of Anatomy; ${ }^{4}$ Department of Biomedicine, Musculoskeletal Research, University of Basel; ${ }^{5}$ Rheumatology, Ultrasound Center, Basel, Switzerland; ${ }^{6}$ Department of Rheumatology, MC Groep Hospitals, Lelystad, Netherlands; ${ }^{7}$ Service de Rhumatologie, Hôpital de la Cavale Blanche, CHRU Brest, Brest, France

Background: In daily practice, surgical biopsy of minor salivary glands is routinely performed for the diagnosis of Sjögren's syndrome. The classification criteria for Sjögren's syndrome imply specific positive labial salivary gland biopsy findings. Surgical biopsies of the minor labial glands may result in up to $6 \%$ of patients in various complications, e.g. numbness of the lower lip. On the other hand, adverse events following core needle biopsies of the parotid gland in nonrheumatological settings were reported as very rare. Even so parotid gland biopsies require a more demanding surgical expertise mainly to protect the facial nerve.

Objectives: The objective of this study was to assess the feasibility and to determine the presence of parotid gland tissue in minimally invasive ultrasound-guided parotid gland biopsies in cadavers performed by rheumatologists using histology as a gold standard.

Methods: Two senior rheumatologists obtained under direct ultrasound visualisation in in-plane technique biopsies of 8 parotid glands from 4 different cadavers using a core biopsy needle (core biopsy needle 18G). One biopsy per gland was taken and was subsequently stored. The direction of the bioptic access is shown in Figure 1. The specimen underwent histological examination by an experienced pathologist. Results: All histological exams showed typical parotid gland tissue. Notably, no facial nerve tissue or major vessels could be detected in the biopsy material.

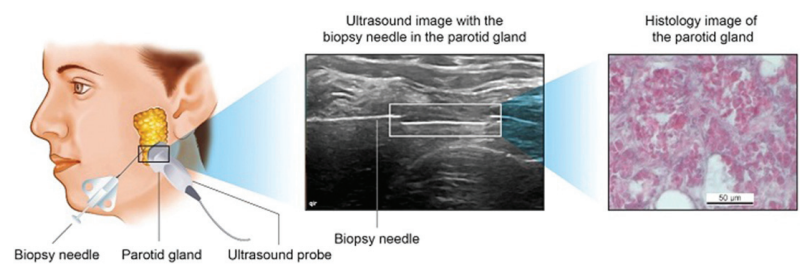

Abstract AB1215 - Figure 1

Conclusions: In this cadaveric feasibility study, we demonstrated that minimally invasive ultrasound guided parotid core biopsy is a highly precise and easy method to obtain salivary gland tissue.

\section{REFERENCES}

[1] Shiboski C. H. et al, 2016 American College of Rheumatology/European League Against Rheumatism Classification Criteria for Primary Sjogren's Syndrome. Arthritis Rheumatol. 2017 Jan;69(1):35-45.

[2] Kim H.J et al, Ultrasound-Guided Core Needle Biopsy in Salivary Glands: A Meta-analysis, Laryngoscope, 00:000-000, 2017.

Disclosure of Interest: None declared DOI: 10.1136/annrheumdis-2018-eular.2578

\section{AB1216 QUANTITATIVE EVALUATION OF THERAPEUTIC CHANGES IN DIGITAL PSORIATIC ARTHRITIS WITH CONTRAST ENHANCED DUAL ENERGY COMPUTED TOMOGRAPHY IODINE MAP}

R. Kawakami ${ }^{1}$, T. Fukuda ${ }^{1}$, S. Ogiwara ${ }^{1}$, M. Momose ${ }^{2}$, T. Tokashiki $^{1}$, Y. Umezawa ${ }^{2}$, A. Asahina ${ }^{2}$, M. Matsushima ${ }^{3}$, H. Nakagawa ${ }^{2}$, K. Fukuda ${ }^{1}$ ${ }^{1}$ Department of Radiology; ${ }^{2}$ Department of Dermatology; ${ }^{3}$ Division of Epidemiology, The Jikei University School of Medicine, Tokyo, Japan

Background: Dual Energy Computed Tomography (DECT) iodine map is highly sensitive in depicting the inflammatory changes of psoriatic arthritis (PsA). A modified PsA Magnetic Resonance Imaging Scoring System (mPsAMRIS) was developed to assess the severity of PsA on DECT iodine map ${ }^{1,2,3}$. DECT iodine map also enables the calculation of iodine uptake in the lesion, which provides a more direct measure of disease activity ${ }^{4}$. However, the usefulness of DECT in evaluating the therapeutic effect of PsA by using iodine quantification, is still undetermined.

Objectives: To assess the validity of DECT iodine map in evaluating the therapeutic effect of PsA by iodine quantification.

Methods: The study included symptomatic PsA patients who underwent two consecutive contrast-enhanced DECT of hand or foot, prior and post medical intervention. All images were independently evaluated by two radiologists. Each symptomatic joint and matched non-symptomatic control joint were scored with mPsAMRIS. To measure the iodine uptake, the region of interest was selected at the level where the joint was most affected. The treatment effect of mPsAMRIS and iodine uptake was calculated by subtracting the results of the matched non symptomatic joints.

Results: The demographics and clinical characteristics of enrolled patients are shown below.

\section{Abstract AB1216 - Table 1}

\section{Demographic and patient Characteristics}

Characteristic

Number of patients

Mean age (yr)

Mean psoriasis duration (yr)

Mean current joint symptom duration (mo)

Fulfilled CASPAR criteria

Mean interval between two DECTs (mo)

$\begin{array}{ccc}\text { All patients } & \text { Men } & \text { Women } \\ 26 & 17 & 9 \\ 50.6(22-89) & 54.2(25-89) & 45.2(22-68) \\ 13.1(0-41) & 12.3(0.5-35) & 14.1(0-41) \\ 26.9(1-192) & 40.3(1-192) & 4.5(1-120) \\ 26 & 17 & 9 \\ 7.0(4-12) & 7(4-12) & 6.4(4-11)\end{array}$

